\title{
The Role of Processing Towards Application of Organic EO Material Modulators
}

\author{
Benjamin C. Olbricht, David L.K. Eng, Stephen T. Kozacik, \\ Shouyuan Shi, and Dennis W. Prather
}

\section{INTRODUCTION}

Polymer electro-optic (EO) modulators have yet to become realized as a widespread commercial success, despite decades of research and many articles detailing superior performance metrics and device demonstrations[1]-[4]. The established technology is a modulator based on the crystalline EO material Lithium Niobate. The systems that employ EO modulators have carefully-designed link budgets which nearly specify a Lithium Niobate modulator, which has demonstrated consistent performance without the pace of performance improvements which make organic materials a volatile- and "moving target[5]." To date there has not been an organic-based modulator that matches each performance metric of a Lithium Niobate modulator, yet offers enhancement to one or more metric. The inability of organic-based modulators to disrupt the established technology has created a climate where organicbased modulators are finding application exclusively in nascent and niche markets. These applications, where systems and link budgets are being regenerated for the performance metrics of organic-based modulators, are offering unprecedented opportunities towards the greater success of photonic systems. Namely, this includes the ability to monolithically integrate hybrid silicon/organic photonic components, the ability to generate spectrally-pure w-band $\mathrm{RF}[6]$, and drastic improvements to the scalable manufacture and CSWaP of photonic devices. In this study, various organic EO materials (OEOMs), claddings, and processing methodologies are presented under the context of nascent and niche applications for EO modulators.

\section{CORE MATERIALS: OEOMS}

An OEOM typically consists of a chromophore, to which the EO response is owed, and a polymer host. The polymer host is generally a transparent polymer that affords the viscosity required in solution to spin-deposit a film of the needed thickness. The polymer also dictates, in many cases, the thermal stability of the material and also influences the linear refractive index as well as the EO activity, as will be presented here. The chromophore used in this study was recently developed and provided for our efforts by Larry Dalton at the University of Washington, Seattle, and is referred to as IKD-150. It is an asymmetrically-substituted polyene bridge that follows the CLD-bridge design with numerous chemical modifications to reduce loss and to increase photostability and EO activity. The dye exhibits a high degree of stability under UV light, which allows the direct UV curing of a cladding material on top of the OEOM. Numerous polymer hosts have been studied and various applications can be envisioned based on the host material of the OEOM. Modulator drive voltage, $V_{\pi}$, a primary performance metric, is commonly expressed in a rearranged form:

$$
n^{3} r_{33}=\frac{d \lambda}{L V_{\pi} \Gamma}
$$

where $n$ is the linear index, $r_{33}$ is the primary element of the proportionality tensor. $d$ is the gap between electrodes of length $L$; the overlap integral $\Gamma$ describes the portion of light confined in the EO material. As such, the figure of merit $n^{3} r_{33}$ isolates each component of the above relationship that is exclusively defined by the material.

IKD-1-50 dispersed in amorphous polycarbonate, the most ubiquitous host for OEOMs offers an $n^{3} r_{33}$ value of approximately $350 \mathrm{pm} / \mathrm{V}$. Polycarbonate is commonly employed for its thermal stability, however some applications, such as photonic links for supercomputing, have operational temperatures of $70 \mathrm{~K}$. Under cryogenic temperatures, thermal stability requirements can be relaxed to achieve drive voltages and power consumption on the order of supercomputer junctions. As such, without tuning fabrication recipes for many TM modulators, methacrylate polymers can be considered despite a $20^{\circ} \mathrm{C}$ depression in the thermal stability compared to polycarbonate hosts. Integrating IKD-1-50 into poly(methyl methacrylate) affords an $n^{3} r_{33}$ value of approximately $600 \mathrm{pm} / \mathrm{V}$. Furthermore, in some TM and many TE-polarized devices, such as slot waveguide modulators[7], the drive voltage can be significantly decreased and the organic EO materials with lower thermal stability values can be considered. Using host polymers such as poly(vinyl butyralco-vinyl alcohol-co-vinyl acetate) the $n^{3} r_{33}$ has been measured to be roughly $750 \mathrm{pm} / \mathrm{V}$. It is also important to note that these materials also have the potential to employ a design that offers an increased modal overlap with a substantially smaller electrode gap due the tighter confinement of radiation in the waveguide core. 
Other applications require increased thermal stability, exceeding the typical Telcordia testing requirements. For these systems, polyimides are commonly used. However, the chromophore used in this study degrades in the presence of certain amines, especially lactams and cyclic amines, even in the solid state.

\section{Claddings}

In the case of a polymer-clad modulator, increasing the overlap integral offers decreased drive voltages, but often competes with an increase in electrode gap unless a lower-index cladding is used. This tradeoff can be broken by utilizing cladding materials to maximize index mismatch with the OEOM core. Fluoropolymers typically offer decreased refractive indices, but often at the expense of wettability. Novel low-index materials are being explored to minimize $\frac{d}{\Gamma}$.

Additionally, the dielectric behavior and strength of a cladding material has strong influence on the processing, namely the "poling," of OEOM modulators. In slab-capacitor devices, such as those characterized by Teng Man Ellipsometry and Attenuated Total Internal Reflection, dielectric layers such as $\mathrm{TiO}_{2}$ were measured to increase the poling efficiency by nearly $40 \%$.

\section{POLING AND PROCESSING}

The bottleneck step of processing OEOMs is a step used to align the dipole moments of the chromophores in the film using an applied DC field and elevated temperatures. This step is commonly referred to as "poling" and is required to accumulate an appreciable EO response in OEOMs. Many device architectures suffer from an inability to efficiently pole the OEOM. The poling process for a UV15-clad IKD-1-50 modulator will be shown and its EO activity agrees remarkably with slab-capacitor devices as measured by attenuated total internal reflection.

Because many materials and devices cannot be poled efficiently, either because of thermal decomposition, delamination, or dielectric breakdown, an array of poling strategies needs to be populated to minimize the design impact of the poling process. For example, laser-assisted poling offers the potential to pole OEOMs more efficiently and to drastically increase $r_{33}$, while decreasing the temperature needed to pole the material by more than $40^{\circ} \mathrm{C}[8]$.

Another notable and recent technique towards poling and processing OEOMs potentially offers the ability to deposit, pole and pattern waveguides in a single step. This process, the solution phase-assisted reorientation of chromophores, or
SPARC, can pole high thermal stability host materials at room temperature using the molecular rotational mobility afforded by the solution phase. This results in a poled OEOM with high thermal stability and without the corresponding tradeoff in EO activity assessed by Langevin models of poling-induced order.

\section{CONCLUSION}

The role of processing dictates the properties and successful integration of OEOMs to an equal extent as chemical synthesis and device design. With redesigned systems, thoughtful processing, and proper molecular and device design, OEOMs will become crucial components of photonic technologies.

\section{ACKNOWLEDGEMENTS}

Ilya Kosilkin and Larry Dalton at the University of Washington, Seattle are recognized for providing the EO chromophore used in this study. Geoff Lindsay is also thanked for many insightful conversations. Gernot Pomrenke (AFOSR) and Rob Nelson (AFRL) are also gratefully acknowledged for providing project support under FA9550-10-1-0363 (AFOSR).

\section{CITATIONS}

[1] Y. Enami, D. Mathine, C. T. DeRose, R. A. Norwood, J. Luo, A. K.-Y. Jen, and N. Peyghambarian, "Hybrid cross-linkable polymer/sol-gel waveguide modulators with $0.65 \mathrm{~V}$ half wave voltage at 1550 nm," Applied Physics Letters, vol. 91, no. 9, p. 093505, 2007.

[2] D. Chen, H. R. Fetterman, A. Chen, W. H. Steier, L. R.

Dalton, W. Wang, and Y. Shi, "Demonstration of $110 \mathrm{GHz}$ electrooptic polymer modulators," Applied Physics Letters, vol. 70, no. 25, pp. 3335-3337, 1997.

[3] Colin V. McLaughlin, Michael L. Hayden, Brent Polishak, Su Huang, Jingdong Luo, Taedong Kim, and Alex K.-Y. Jen,

"Wideband 15-THz response using organic electrooptic emitter-sensor pairs at telecommunications wavelengths," Appl. Phys. Lett., vol. 92, pp. $151107-1,2008$

[4] "GigOptix Polymer Modulators have passed Telcordia Testing, http://www.gigoptix.com/images/TFPSTelcordiaUpdate.pdf." [5] L. R. Dalton, P. A. Sullivan, and D. H. Bale, "Electric field poled organic electro-optic materials: state of the art and future prospects," Chemical Reviews, vol. 110, p. 25, 2010.

[6] G. J. Schneider, J. A. Murakowski, C. A. Schuetz, S. Shi, and D. W. Prather, "Radiofrequency signal-generation system with over seven octaves of continuous tuning," Nat Photon, vol. 7, no. 2, pp. 118-122, Feb. 2013.

[7] S. Shi and D. W. Prather, "Ultrabroadband Electro-Optic Modulator Based on Hybrid Silicon-Polymer Dual Vertical Slot Waveguide," Advances in OptoElectronics, vol. 2011.

[8] B. C. Olbricht, P. A. Sullivan, G.-A. Wen, A. A. Mistry, J. A. Davies, T. R. Ewy, B. E. Eichinger, B. H. Robinson, P. J. Reid, and L. R. Dalton, "Laser-Assisted Poling of Binary Chromophore Materials," J. Phys. Chem. C, vol. 112, no. 21, pp. 7983-7988, May 2008. 